\title{
Potential theoretic approach to rendezvous numbers
}

\author{
Bálint Farkas, Szilárd Gy. Révész \\ Alfréd Rényi Institute of Mathematics \\ Hungarian Academy of Sciences \\ Reáltanoda utca 13-15, H-1053, Budapest, Hungary \\ revesz@renyi.hu \\ Technische Universität Darmstadt \\ Fachbereich Mathematik, AG4 \\ Schloßgartenstraße 7, D-64289 Darmstadt, Germany \\ farkas@mathematik.tu-darmstadt.de
}

\begin{abstract}
We analyze relations between various forms of energies (reciprocal capacities), the transfinite diameter, various Chebyshev constants and the so-called rendezvous or average number. The latter is originally defined for compact connected metric spaces $(X, d)$ as the (in this case unique) nonnegative real number $r$ with the property that for arbitrary finite point systems $\left\{x_{1}, \ldots, x_{n}\right\} \subset X$, there exists some point $x \in X$ with the average of the distances $d\left(x, x_{j}\right)$ being exactly $r$. Existence of such a miraculous number has fascinated many people; its normalized version was even named "the magic number" of the metric space. Exploring related notions of general potential theory, as set up, e.g., in the fundamental works of Fuglede and Ohtsuka, we present an alternative, potential theoretic approach to rendezvous numbers.
\end{abstract}

AMS Subj. Clas.(2000): Primary: 31C15. Secondary: 28A12, 54D45

Keywords and phrases: Locally compact Hausdorff topological spaces, potential theoretic kernel function in the sense of Fuglede, potential of a measure, energy integral, energy and capacity of a set, transfinite diameter, Chebyshev constant, weak*-topology, (weak) rendezvous number, average distance, minimax theorem.

\section{Introduction}

Rendezvous numbers, studied usually in metric spaces, have already attracted much attention. The existence and uniqueness of such a miraculous number in compact connected metric spaces was shown by O. Gross [17]. Later many authors were fascinated

Supported by OTKA (Hungarian Scientific Research Fund) 
by the topic. They calculated the rendezvous numbers in particular cases, and extended the results in the direction of weak rendezvous numbers or rendezvous numbers of unit spheres in Banach spaces (see, e.g., [2], [4], [7], [16], [21], [22], [24], [35], [36], [37, 38, 39] and $[40])$.

Already Björck applied certain tools of potential theory in studying constants related to rendezvous numbers [4]. Now, exploring notions of general, abstract potential theory, as set up, e.g., in the fundamental works of Fuglede and Ohtsuka - and, in particular, drawing from some exploration of mutual energies, Chebyshev constants and transfinite diameters over locally compact topological spaces with lower semicontinuous, nonnegative and symmetric kernels (see [15], [26], [8]) - we arrive at an understanding of these quantities from a more general viewpoint.

However, to achieve this, we need to recover and even partially extend the relevant basic material. In particular, we thoroughly investigate energies and Chebyshev constants, and even also their "minimax duals" in function of two sets. The technical reason for that is that the classical definitions are kind of saddle point special cases of these more general notions, and we need to utilize special monotonicity and other properties, which stay hidden when considering only the diagonal cases.

Let us recall the appropriate setting of potential theory in locally compact spaces. First, $+\infty$ is added to the set of real numbers, i.e., we let $\overline{\mathbb{R}}:=\mathbb{R} \cup\{+\infty\}$ endowed with its natural topology such that $\overline{\mathbb{R}}_{+}$will be compact. Moreover, we will use the notation conv $E$ for the convex hull of a subset $E \subset \mathbb{R}$ and $\overline{\text { conv }} E$ for the closed convex hull in $\overline{\mathbb{R}}_{+}$, meaning, for example, $\overline{\operatorname{conv}}(0,+\infty)=[0,+\infty]$.

Throughout the paper $X$ denotes a locally compact Hausdorff space, and $k: X \times$ $X \rightarrow \overline{\mathbb{R}}$ is a kernel function in the sense of Fuglede [14, p. 149]. That is, we assume that $k$ is lower semicontinuous (l.s.c.) as a two variable function over $X \times X$, and that $-\infty<k(x, y) \leq+\infty$. Moreover, in this paper we assume that $k \geq 0$, and that $k$ is symmetric, i.e., $k(x, y)=k(y, x)$ for all $x, y \in X$.

Under these standard assumptions there exist energy-minimizing (or, as sometimes called, capacitary) measures (see, e.g., [14, Theorem 2.3]), and also the equivalence of the energies (reciprocal capacities) follows, see [14, p. 159].

The vector space of all finite, signed regular Borel measures on $X$ is $\mathfrak{M}^{ \pm}(X):=$ $\mathfrak{M}(X)-\mathfrak{M}(X)$, where

$$
\mathfrak{M}:=\mathfrak{M}(X):=\{\mu: \mu \text { is a positive, regular Borel measure on } X,\|\mu\|<\infty\} .
$$

We will also denote by $\mu$ the Carathèodory extension of $\mu$ to an outer measure, while $\mu$-measurability of a set $H$ refers to the usual Carathèodory measurability. Also, let $\mathfrak{M}_{1}(X)$ be the set of probability measures from $\mathfrak{M}(X), \mathfrak{M}_{1}:=\mathfrak{M}_{1}(X):=\{\mu \in \mathfrak{M}(X)$ : $\mu(X)=1\}$. We say that $\mu$ is concentrated on a set $H \subset X$, if each compact set intersects the complement $X \backslash H$ of $H$ in a set of zero (outer-) measure. In our case all measures are finite, hence this is the same as requiring measurability and having full measure, cf. [14, p. 146]. Furthermore, for an arbitrary set $H \subset X$, let $\mathfrak{M}_{1}(H):=\left\{\mu \in \mathfrak{M}_{1}\right.$ : $\mu$ is concentrated on $H$ \}. We also say that $\mu$ is supported in $H$ if $\operatorname{supp} \mu$ is a subset of $H$. Note that, if $H$ is a closed set, then the two notions - concentrated and supported on $H$ - are the same. For convenience we introduce the notation $\mathfrak{M}_{1}^{\#}(H)$ for finitely supported probability measures on $H$. 
The customary topology on $\mathfrak{M}$ (or on $\mathfrak{M}^{ \pm}$) is the vague topology which is the locally convex topology determined by the seminorms $\mu \mapsto\left|\int_{X} f \mathrm{~d} \mu\right|, f \in C_{c}(X)$, where $C_{c}(X)$ denotes the space of continuous functions with compact support. In most places we consider only the family $\mathfrak{M}_{1}(K)$ of probability measures supported on the same compact set $K$. In this case, by the Riesz Representation Theorem, $\mathfrak{M}^{ \pm}(K)=C(K)^{\prime}$, moreover, the weak*-topology determined by $C(K)$ and the vague topology coincide. We will use nets to describe convergence in $X$ or in $\mathfrak{M}$. For example, we will use the filtering family $\mathcal{K}$ (directed upwards) of all compact subsets of $X$. Given $\mu \in \mathfrak{M}(X)$ we define its traces $\mu_{K}$ on compact $\operatorname{sets}^{1} K \Subset X$ by $\mu_{K}(A):=\mu(A \cap K)$. Using the above terminology the regularity of $\mu$ implies that $\lim _{\mathcal{K}} \mu_{K}=\mu$ in the vague topology. This, among others, means that $\lim _{\mathcal{K}}\left\|\mu_{K}\right\|=\|\mu\|$.

The potential and energy of $\mu \in \mathfrak{M}$ are defined as

$$
U^{\mu}(x):=\int_{X} k(x, y) \mathrm{d} \mu(y), \quad W(\mu):=\int_{X} \int_{X} k(x, y) \mathrm{d} \mu(y) \mathrm{d} \mu(x) .
$$

The existence of these two integrals follow because $k \geq 0$ and it is lower semicontinuous - however, $W(\mu)$ and $U^{\mu}$ may attain $+\infty$ as well.

There are three different definitions of energy (reciprocal capacity) in use in the fundamental work of Fuglede, see [14, p. 150], [14, Eq. (1) and (2), p. 153]. All these are related to the supremum of the potential $U^{\mu}(x)$ over particular subsets of $X$.

In some less widely known works, however the theory have already been extended to generalized mutual energies and "dual energies" of two sets as variables, see [15] and [26]: moreover, even generalized, two-variate Chebyshev constants were defined in [26] as well. We add here the notion of dual Chebyshev constants, too. These allow to present a fairly general approach to rendezvous numbers.

We try to make this paper as self-contained, as possible. In particular, we use only a basic familiarity with the monographic paper of Fuglede [14], which we consider and use as our basic reference to potential theory in general. For all material above those directly quoted from [14] we give a precise description, or at least sketch a proof. Nevertheless, for historical completeness we will refer to various potential theoretical papers and many potential theoretical results throughout.

\section{Definitions and preliminaries}

For our purposes we consider the following notion of "energy", among whose special cases will be the three quantities used in [14, p. 150-153] (and recalled also in Definition 2.2 below).

Definition 2.1. Let $H \subset X$ be fixed, and $\mu \in \mathfrak{M}_{1}(X)$ be arbitrary. First put

$$
Q(\mu ; H):=\sup _{x \in H} U^{\mu}(x), \quad \text { and also } \quad \underline{Q}(\mu ; H):=\inf _{x \in H} U^{\mu}(x) .
$$

\footnotetext{
${ }^{1}$ Here and also throughout the sequel, the notation $K \Subset H$ means that $K$ is a compact subset of $H$.
} 
The quasi-uniform energy and the restricted quasi-uniform energy of $H$ are

$$
q(H):=\inf _{\mu \in \mathfrak{M}_{1}(H)} Q(\mu ; H) \quad \text { and } \quad q^{\#}(H):=\inf _{\mu \in \mathfrak{M}_{1}^{*}(H)} Q(\mu ; H) .
$$

Moreover, we define analogously for any two sets $H, L \subset X$ the quantities

$$
q(H, L):=\inf _{\mu \in \mathfrak{M}_{1}(H)} Q(\mu ; L) \text { and } q^{\#}(H, L):=\inf _{\mu \in \mathfrak{M}_{1}^{\#}(H)} Q(\mu ; L) .
$$

Furthermore, let us recall the dual notions [26]

$$
\underline{q}(H, L):=\sup _{\nu \in \mathfrak{M}_{1}(H)} \underline{Q}(\nu ; L) \quad \text { and } \quad \underline{q}^{\#}(H, L):=\sup _{\nu \in \mathfrak{M}_{1}^{\#}(H)} \underline{Q}(\nu ; L),
$$

and, similarly to $(2.2)$

$$
\underline{q}(H):=\underline{q}(H, H) \quad \text { and } \quad \underline{q}^{\#}(H):=\underline{q}^{\#}(H, H) .
$$

These extended definitions - with different notation - appear already in the work of Fuglede [15] and Ohtsuka [26]. Recall the following univariate versions of energies, commonly used in linear potential theory.

Definition 2.2. a) Let $\mu \in \mathfrak{M}_{1}$ be any measure. Then we write

$$
\begin{aligned}
& U(\mu):=\sup _{x \in X} U^{\mu}(x)=\sup _{K \Subset X} U\left(\mu_{K}\right), \\
& V(\mu):=\sup _{x \in \operatorname{supp} \mu} U^{\mu}(x)=\sup _{K \Subset X} V\left(\mu_{K}\right),
\end{aligned}
$$

the second forms, as well as an analogous statement for $W(\mu)$, following from [14, Lemma 2.2.2 and Footnote 1 on p. 152].

b) Accordingly, the "uniform", "de la Vallée-Poussin" and "Wiener" energies (reciprocal capacities) of any set $H \subset X$ are

$$
\begin{aligned}
u(H) & :=\inf _{\mu \in \mathfrak{M}_{1}(H)} U(\mu)=\inf _{\mu \in \mathfrak{M}_{1}(H), \operatorname{supp} \mu \Subset H} U(\mu)=\inf _{K \Subset H} u(K), \\
v(H) & :=\inf _{\mu \in \mathfrak{M}_{1}(H)} V(\mu)=\inf _{\mu \in \mathfrak{M}_{1}(H), \operatorname{supp} \mu \Subset H} V(\mu)=\inf _{K \Subset H} v(K), \\
w(H) & :=\inf _{\mu \in \mathfrak{M}_{1}(H)} W(\mu)=\inf _{\mu \in \mathfrak{M}_{1}(H), \operatorname{supp} \mu \Subset H} W(\mu)=\inf _{K \Subset H} w(K),
\end{aligned}
$$

respectively, where equivalence of the last forms can be proved based on [14]. Instead of doing that, we will prove the similar, but more general statement for $q$ below in Lemma 2.4 .

Lemma 2.3. Let $H, L \subset X$. Equipping the set $\mathfrak{M}_{1}(H)$ with the vague topology the following functions are lower semicontinuous

a)

$$
\mathfrak{M}_{1}(H) \times L \ni(\mu, x) \mapsto U^{\mu}(x)\left(=\int_{X} k(x, y) \mathrm{d} \mu(y)\right)
$$




$$
\begin{aligned}
& \mathfrak{M}_{1}(H) \times \mathfrak{M}_{1}(L) \ni(\mu, \nu) \mapsto W(\mu, \nu):=\int_{X} \int_{X} k(x, y) \mathrm{d} \mu(y) \mathrm{d} \nu(x) \\
& \mathfrak{M}_{1}(H) \ni \mu \mapsto Q(\mu ; L)\left(=\sup _{x \in L} U^{\mu}(x)\right)
\end{aligned}
$$

Proof. Part a) and b) are taken from [14, Lemma 2.2.1], while part c) follows from part a) by noticing that $Q(\mu ; L)$ is a pointwise supremum of l.s.c. functions.

Lemma 2.4. For any two sets $H, L \subset X$ we have

$$
\begin{aligned}
q(H, L) & =\inf _{\substack{\mu \in \mathfrak{M}_{1}(H) \\
\operatorname{supp} \mu \Subset H}} Q(\mu ; L)=\inf _{K \Subset H} q(K, L), \\
q^{\#}(H, L) & =\inf _{\mu \in \mathfrak{M}_{1}^{\#}(H)} Q(\mu ; L)=\inf _{K \Subset H} q^{\#}(K, L) .
\end{aligned}
$$

Proof. First,

$$
q(H, L):=\inf _{\mu \in \mathfrak{M}_{1}(H)} Q(\mu ; L) \leq \inf _{\substack{\mu \in \mathfrak{M}_{1}(H) \\ \operatorname{supp} \mu \Subset H}} Q(\mu ; L)=\inf _{K \Subset H} q(K ; L)
$$

is obvious. Second, for any measure $\mu \in \mathfrak{M}_{1}(H)$ and compact set $K \Subset H$ we have $Q(\mu ; L) \geq Q\left(\mu_{K} ; L\right)=\left\|\mu_{K}\right\| Q\left(\nu_{\mu, K} ; L\right)$ with $\nu_{\mu, K}:=\mu_{K} /\left\|\mu_{K}\right\|$ now satisfying $\nu_{\mu, K} \in$ $\mathfrak{M}_{1}(K)$. Plainly, for any measure $\mu \in \mathfrak{M}_{1}(X)$ we have $\mu_{K} \leq \mu$, and thus for nonnegative kernels $k$ also the potentials satisfy $U^{\mu_{K}} \leq U^{\mu}$. Now let us select some sequence of increasing compact sets $K_{n} \Subset H$ with $\mu\left(K_{n}\right)>1-\frac{1}{n}$. Such a sequence exists by regularity of the measure $\mu$, since $\mu \in \mathfrak{M}_{1}(H)$ entails that the set $H$ is $\mu$-measurable (cf. [14, p. 146]). Thus we find

$$
\inf _{K \Subset H} q(K, L) \leq q\left(K_{n}, L\right) \leq Q\left(\nu_{\mu, K_{n}} ; L\right) \leq \frac{1}{1-1 / n} Q\left(\mu_{K_{n}} ; L\right) \leq \frac{1}{1-1 / n} Q(\mu ; L),
$$

for all $n \geq 1$ and hence $\inf _{K \Subset H} q(K, L) \leq Q(\mu ; L)$. Since here $\mu \in \mathfrak{M}_{1}(H)$ is arbitrary, we obtain $\inf _{K \Subset H} q(K, L) \leq q(H, L)$. On combining this with (2.13) gives the last two formulations of (2.11). The proof of (2.12) is similar.

Remark 2.5. Although one would like to have $q(H)=\inf _{K \Subset H} q(K)$, this is false in general.

Example 2.6. As an example one can consider a two point metric space $X=\{a, b\}$ with the discrete metric as the kernel $k$, and take $H=X$. It is obvious that for $\# K=1$ we have $q(K)=0$, while $Q(\mu ; H)=\max (\mu(a), \mu(b)) \geq 1 / 2$ for any $\mu \in \mathfrak{M}_{1}$, hence $q(H)=1 / 2 \supsetneqq \inf _{K \Subset H} q(K)=0$. The reason for the occurrence of this difficulty is lack of monotonicity of $q(H)$. On the other hand, fixing one variable of the two-set function $q$, the functions $q(\cdot, L)$ and $q(H, \cdot)$ are monotonous (see also Lemma 3.5 below). That is why involving two-set functions is necessary here and throughout the paper.

Not surprisingly, a kind of a dual statement holds for $\underline{q}$, at least for compact $L$. 
Lemma 2.7. Let $H \subset X$ be arbitrary and $L \Subset X$ be compact. Then we have

$$
\begin{aligned}
\underline{q}(H, L) & =\sup _{\substack{\mu \in \mathfrak{M}_{1}(H) \\
\operatorname{supp} \mu \Subset H}} \underline{Q}(\mu ; L)=\sup _{K \Subset H} \underline{q}(K, L), \\
\underline{q}^{\#}(H, L) & =\sup _{\substack{\mu \in \mathfrak{M}_{1}^{\#}(H) \\
\text { supp } \mu \Subset H}} \underline{Q}(\mu ; L)=\sup _{K \Subset H} \underline{q}^{\#}(K, L) .
\end{aligned}
$$

Proof. The inequality

$$
\underline{q}(H, L):=\sup _{\mu \in \mathfrak{M}_{1}(H)} \underline{Q}(\mu ; L) \geq \sup _{\substack{\mu \in \mathfrak{M}_{1}(H) \\ \operatorname{supp} \mu \Subset H}} \underline{Q}(\mu ; L)=\sup _{K \Subset H} \underline{q}(K ; L)
$$

is trivial. Similarly to the proof of Lemma 2.4 let $\mu \in \mathfrak{M}_{1}(H)$ be arbitrary and consider its traces $\mu_{K}$ on compact sets $K$. Further define $\nu_{K}:=\nu_{\mu, K}:=\mu_{K} /\left\|\mu_{K}\right\|$. (Note that $\left\|\mu_{K}\right\|>0$ can be assumed by fixing some compact set $\widetilde{K} \Subset H$ with $\mu(\widetilde{K})>0$ and considering only compact sets $K \supset \widetilde{K}$ here.) Then clearly $\nu_{K} \in \mathfrak{M}_{1}(K)$. By lower semicontinuity of $U^{\nu_{K}}$ (cf. Lemma 2.3 a)) and referring to compactness of $L$ we find an element $y_{K} \in L$ minimizing $U^{\nu_{K}}(y)$ on $L$. Because $\mu$ is a regular Borel measure $\lim _{K \in \mathcal{K}} \nu_{K}=\mu$ in the vague topology.

Also, by compactness of $L$ we have a subnet $\mathcal{N}$ of $\mathcal{K}$ such that $\lim _{K \in \mathcal{N}} y_{K}=y_{0}$ for some $y_{0}$. Again by l.s.c. we obtain (using $\nu_{K} \rightarrow \mu$ and $y_{K} \rightarrow y_{0}$ along $\mathcal{N}$, and referring to Lemma 2.3 a)),

$$
\liminf _{\mathcal{N}} U^{\nu_{K}}\left(y_{K}\right) \geq U^{\mu}\left(y_{0}\right) \geq \inf _{y \in L} U^{\mu}(y)=\underline{Q}(\mu ; L) .
$$

Summing up

$$
\sup _{K \Subset H} \underline{q}(K, L) \geq \liminf _{\mathcal{N}} \inf _{L} U^{\nu_{K}}=\liminf _{\mathcal{N}} U^{\nu_{K}}\left(y_{K}\right) \geq \underline{Q}(\mu ; L) .
$$

Taking supremum in $\mu$ and comparing to (2.17) yields the assertion. The proof of (2.16) is analogous.

The quantities of $n^{\text {th }}$ diameters, transfinite diameters and Chebyshev constants were generalized from the classical logarithmic kernel case to some more general kernels already by Pólya, Szegö [30], Carleson [5] and Choquet [6] the way we present below. (Note that another direction of generalization, due to Zaharjuta [41], is also considered in $\mathbb{C}^{n}$.)

Definition 2.8. Let $H \subset X$ be fixed. The $n^{\text {th }}$ diameter of $H$ is defined as follows.

$$
D_{n}(H):=\inf _{w_{1}, \ldots, w_{n} \in H} \frac{2}{(n-1) n}\left(\sum_{1 \leq j<l \leq n} k\left(w_{j}, w_{l}\right)\right) .
$$

Definition 2.9. For an arbitrary $H \subset X$ the $n^{\text {th }}$ Chebyshev constant of $H$ is defined as

$$
M_{n}(H):=\sup _{w_{1}, \ldots, w_{n} \in H} \inf _{x \in H} \frac{1}{n}\left(\sum_{k=1}^{n} k\left(x, w_{k}\right)\right) .
$$


In fact, even in the classical literature another variant of the Chebyshev constant occurs. Namely, for an arbitrary $H \subset X$ the modified $n^{\text {th }}$ Chebyshev constant of $H$ is defined as

$$
C_{n}(H):=\sup _{w_{1}, \ldots, w_{n} \in X} \inf _{x \in H} \frac{1}{n}\left(\sum_{k=1}^{n} k\left(x, w_{k}\right)\right),
$$

that is, allowing the "zeroes" $w_{j}$ spread out in $X$, but considering the values only on $H$.

To put these Chebyshev constants into a general framework was probably a reason why Ohtsuka considered the following notion [26]. Our motivation is mentioned already in Remark 2.5 and will be even clearer in view of Proposition 4.5.

Definition 2.10. For arbitrary $H, L \subset X$ the (general) $n^{\text {th }}$ Chebyshev constant of $L$ with respect to $H$ is defined as

$$
M_{n}(H, L):=\sup _{w_{1}, \ldots, w_{n} \in H} \inf _{x \in L} \frac{1}{n}\left(\sum_{k=1}^{n} k\left(x, w_{k}\right)\right) .
$$

Remark that Ohtsuka [26] defines these quantities essentially without any assumption on the kernel $k$. To prove the convergence of these sequences one has to assume that the kernel does not take the value $-\infty$, then on compact set an l.s.c. kernel will be bounded from below. The symmetry assumption - here - does not play any role.

Finally, let us define - in a slightly more general setting, that is, forgetting about the metric, usually involved in the context - the (weak) rendezvous number(s), or average distance number(s) of the space $X$, or even of subsets of $X$. Again, for good reasons we define these notions in dependence of two sets as variables.

Definition 2.11. For arbitrary subsets $H, L \subset X$ the $n^{\text {th }}$ (weak) rendezvous set of $L$ with respect to $H$ is

$$
R_{n}(H, L):=\bigcap_{w_{1}, \ldots, w_{n} \in H} \overline{\operatorname{conv}}\left\{p_{n}(x):=\frac{1}{n} \sum_{j=1}^{n} k\left(x, w_{j}\right) \quad: \quad x \in L\right\} .
$$

Correspondingly, one defines

$$
R(H, L):=\bigcap_{n=1}^{\infty} R_{n}(H, L), \quad R(H):=R(H, H) .
$$

Similarly, one defines the (weak) average set of $L$ with respect to $H$ as

$$
A(H, L):=\bigcap_{\mu \in \mathfrak{M}_{1}(H)} \overline{\operatorname{conv}}\left\{U^{\mu}(x) \quad: \quad x \in L\right\}, \quad A(H):=A(H, H) .
$$

Remark 2.12. Denoting the interval

$$
A(\mu, L):=[\underline{Q}(\mu ; L), Q(\mu ; L)]=\overline{\operatorname{conv}}\left\{U^{\mu}(x): x \in L\right\},
$$

we see that $R_{n}(H, L), R(H, L)$ and $A(H, L)$ are all of the form $\bigcap_{\mu} A(\mu, H)$, with $\mu$ ranging over all averages of $n$ Dirac measures at points of $H$, over all probability measures finitely supported in $H$ (and of rational probabilities, but compare to Lemma 3.9 below) and over all of $\mathfrak{M}_{1}(H)$, respectively. 
Remark 2.13. If $k$ is a continuous kernel - in particular when it is a metric on $X$,then it suffices to take convex hull instead of closed convex hull whenever $L$ is compact, since then together with $k$ also $U^{\mu}(x)$ is continuous for any probability measure $\mu$. Thus for compact subsets $L$ of metric spaces a real number $r \in \mathbb{R}_{+}$belongs to $R(H, L)$ if and only if for any finite system of (not necessarily distinct) points $x_{1}, \ldots, x_{n} \in H$ (of number $n \in \mathbb{N}$ taken arbitrarily) we always have points $y, z \in L$ satisfying

$$
\frac{1}{n} \sum_{j=1}^{n} k\left(y, x_{j}\right) \leq r \quad \text { and } \quad \frac{1}{n} \sum_{j=1}^{n} k\left(z, x_{j}\right) \geq r,
$$

which is the usual definition of weak rendezvous numbers in metric spaces (see [36]). Moreover, in case the set $L$ is connected, this is further equivalent to the existence of a "rendezvous point" $x \in L$ with

$$
\frac{1}{n} \sum_{j=1}^{n} k\left(x, x_{j}\right)=r
$$

In particular, for compact and connected $L$ in a metric space (or in a locally compact space with continuous kernel $k$ ) the rendezvous set $R(H, L)$ consists of a unique point, say $R(H, L)=\{r(H, L)\}$, if this latter property is satisfied only for $r=r(H, L)$.

Remark 2.14. If $k$ is only l.s.c., also potentials are l.s.c., which entails that they take their infimum over compact sets. Thus for compact $L$ the first half of the above equivalent formulation (2.23) remains valid even for general kernels. However, for the second part we must already write that " $\forall s<r \exists z \in L$ such that $\frac{1}{n} \sum_{j=1}^{n} k\left(z, x_{j}\right)>s$ ". Such modification of the formulation is necessary also when we consider sets $L \subset X$ which are not compact, or when we are discussing the case when $+\infty \in R(H, L)$. Clearly, in our settings $R_{n}(H, L), R(H, L)$ and $A(H, L)$ are subsets of $[0, \infty]$, but note that traditionally rendezvous numbers or average numbers are considered only among the reals. Hence even in metric spaces our notions slightly differ from the usual ones regarding the role of $+\infty$.

Example 2.15. For example we say that even $+\infty \in R(H, L)$ if for all finite systems of (not necessarily distinct) points $x_{1}, \ldots, x_{n} \in H$ and for all real $s$, however large, there is $z \in L$ satisfying $\frac{1}{n} \sum_{j=1}^{n} k\left(z, x_{j}\right)>s$. Thus, e.g., taking $X:=\mathbb{R}$ with the usual Euclidean metric $k(x, y):=|x-y|$, the four possible variations with the sets $\mathbb{R}$ and $I:=[0,1]$ yield $R(\mathbb{R}, I)=\emptyset, R(I, \mathbb{R})=[1 / 2, \infty], R(I):=R(I, I)=\{1 / 2\}$ (and thus $r(I)=1 / 2$ exists uniquely; see [7]) and $R(\mathbb{R}):=R(\mathbb{R}, \mathbb{R})=\{+\infty\}$, see Proposition 4.3. Note that this last case classically would be interpreted as a case when there is no rendezvous number, while in our notation this is a case of uniqueness with $r(\mathbb{R})=+\infty$. Similar phenomena occur also in case of finite accumulation points not belonging to the "restricted sense rendezvous sets", interpreted without closure.

The aim of the present work is to study properties of these sets and set functions. First we look at the various energies (reciprocal capacities) and Chebyshev constants associated to a set $H \subset X$. 


\section{Basic properties of the quantities defined}

Most of the results in this section were already obtained by Fuglede [15] and Ohtsuka [26]. To be self-contained we present full proofs to them.

Let us start by recalling from [6] and [26] the definition of the Chebyshev constant and the transfinite diameter as the limit of the respective sequences. It is shown in [6] that the sequence of $n^{\text {th }}$ diameters is monotonically increasing (cf. [12] or [31] for the classical case; see also $[5,8])$. The limit is denoted by $D(H):=\lim _{n \rightarrow \infty} D_{n}(H)$ and is called the transfinite diameter of $H$.

To prove the convergence of the sequence of $n^{\text {th }}$ Chebyshev constants the following lemma can be used. (See [12, p. 233]. See also [31], part I, Ch. 3, §1, Problem 98, p. 23 \& p. 198 or [8].)

Lemma 3.1 (Fekete). Let $\left(s_{n}\right)$ be a quasi-monotonous sequence of real numbers, meaning that either $(n+m) s_{n+m} \leq n s_{n}+m s_{m}$ (in this case we say that $\left(s_{n}\right)$ is quasi-monotonically decreasing) or $(n+m) s_{n+m} \geq n s_{n}+m s_{m}$ (the sequence $\left(s_{n}\right)$ is quasi-monotonically increasing). Then $\lim _{n \rightarrow \infty} s_{n}=\inf s_{n}$ (when $\left(s_{n}\right)$ is quasi-monotonically decreasing) or $\lim _{n \rightarrow \infty} s_{n}=\sup s_{n}$ (when $\left(s_{n}\right)$ is quasi-monotonically increasing), the inf (sup) being either finite or $-\infty(+\infty)$.

Proposition 3.2. For any $H, L \subset X$, the Chebyshev constants $M_{n}(H, L)$ converge, more precisely

$$
\sup _{n \in \mathbb{N}} M_{n}(H, L)=\lim _{n \rightarrow \infty} M_{n}(H, L) .
$$

In particular, $\sup _{n \in \mathbb{N}} M_{n}(H, H)=\lim _{n \rightarrow \infty} M_{n}(H, H)$ and $\sup _{n \in \mathbb{N}} M_{n}(X, H)=\lim _{n \rightarrow \infty} M_{n}(X, H)$.

Proof. Since $M_{n}(H, L)$ is quasi-monotone increasing, once $M_{n}(H, L)=+\infty$ holds, the subsequent terms are all infinity as well, i.e., $M_{n+1}(H, L)=+\infty$. Hence $M_{n}(H, L)$ converges (in the extended sense) to $+\infty$. If $M_{n}(H, L)$ is finite for all $n \in \mathbb{N}$ Lemma 3.1 applies. For the classical Chebyshev constants themselves see [12, p. 233] or [31], part I, Ch. 3, §1, Problem 98, p. 23 \& p. 198 or [8]. For more general kernels and of a similar notation (with respect of taking logarithms of classical formulations) see [5, Chapter IV].

The limit $M(H, L):=\lim _{n \rightarrow \infty} M_{n}(H, L)$ is called the general Chebyshev constant of $L$ relative to $H$. In the two special cases the terminology for the limit is the following: $M(H):=\lim _{n \rightarrow \infty} M_{n}(H)$ is called the Chebyshev constant of $H$ and $C(H):=\lim _{n \rightarrow \infty} C_{n}(H)$ is the modified Chebyshev constant.

The following result on the relation of $M$ and $D$ was shown in [8], but follows also from the combination of [6] and [26].

Proposition 3.3. For any $n \in \mathbb{N}$ and $H \subset X$ we have $D_{n}(H) \leq M_{n}(H)$, and thus also $D(H) \leq M(H)$. [8].

Let us recall the connection of the transfinite diameter $D$ and the energy $w$ from $[6]$,

Theorem 3.4. The following assertions hold for $D$ and $w$. 
a) $D(H) \leq w(H)$ for all $H \subset X$.

b) $D(K)=w(K)$ for all $K \Subset X$ compact sets.

c) If the kernel $k$ is finite-valued (i.e., $0 \leq k<+\infty)$, then $D(H)=w(H)$ for all $H \subset X$.

The following proposition is trivial from the definitions, but should be stated explicitly here.

Lemma 3.5. Let $X$ be a locally compact Hausdorff space and $k$ be any nonnegative, l.s.c., symmetric kernel on $X$ and $L \subset X$ fixed. Then the set functions

$$
\begin{gathered}
H \mapsto u(H), v(H), w(H), q(H, L), q^{\#}(H, L), \underline{q}(L, H), \underline{q}^{\#}(L, H), \\
D_{n}(H), D(H), M_{n}(L, H) \text { and } M(L, H) \in \overline{\mathbb{R}}
\end{gathered}
$$

are non-increasing. Also, the set-to-set functions

$$
H \mapsto R_{n}(H, L), R(H, L) \text { and } A(H, L) \subset \overline{\mathbb{R}}_{+}
$$

are non-increasing. On the other hand for fixed $H \subset X$ the functions

$$
L \mapsto q(H, L), q^{\#}(H, L), \underline{q}(L, H), \underline{q}^{\#}(L, H), M_{n}(L, H) \text { and } M(L, H) \in \overline{\mathbb{R}}_{+}
$$

and also the set-to-set functions

$$
L \mapsto R_{n}(H, L), R(H, L) \text { and } A(H, L) \subset \overline{\mathbb{R}}_{+}
$$

are non-decreasing.

Proof. Trivial by observation of the definitions. Allowing infima or suprema over larger sets results in smaller or larger values, respectively.

Lemma 3.6. Let $H, L \subset X$ be arbitrary. Then we have $\underline{q}(H, L) \leq q(L, H)$.

Proof. Let $L^{\prime} \subseteq L$ compact and $\mu \in \mathfrak{M}_{1}(H), \nu \in \mathfrak{M}_{1}\left(L^{\prime}\right)$, both compactly supported in $H$ and $L^{\prime}$, respectively. By Fubini's theorem we can write

$$
\begin{aligned}
\underline{Q}\left(\mu ; L^{\prime}\right) & =\inf _{x \in L^{\prime}} U^{\mu}(x)=\inf _{x \in L^{\prime}} \int_{\operatorname{supp} \mu} k(x, y) \mathrm{d} \mu(y) \leq \int_{L^{\prime}} \int_{\operatorname{supp} \mu} k(x, y) \mathrm{d} \mu(y) \mathrm{d} \nu(x)= \\
& =\int_{\operatorname{supp} \mu} \int_{L^{\prime}} k(x, y) \mathrm{d} \nu(x) \mathrm{d} \mu(y) \leq \sup _{y \in H} \int_{L^{\prime}} k(x, y) \mathrm{d} \nu(x)= \\
& =\sup _{y \in H} U^{\nu}(y)=Q(\nu ; H) .
\end{aligned}
$$

Taking supremum and infimum over $\mu$ and $\nu$ respectively it follows, also by applying Lemma 2.4 and 2.7, that

$$
\underline{q}\left(H, L^{\prime}\right) \leq q\left(L^{\prime}, H\right) .
$$

So by Lemma 3.5 and taking infimum for $L^{\prime}$ with the use of Lemma 2.4 again, we obtain

$$
\underline{q}(H, L) \leq \inf _{L^{\prime} \Subset L} q\left(L^{\prime}, H\right)=q(L, H),
$$

hence the assertion. 
Lemma 3.6 and the trivial assertions in Lemma 3.5 have the following corollary.

Corollary 3.7. Let $H \subset L \subset X$ be arbitrary subsets. Then we have $\underline{q}(H, L) \leq q(H, L)$.

Proof. In view of Lemma 3.6 we have both $\underline{q}(H, L) \leq q(L, H)$ and $\underline{q}(L, H) \leq q(H, L)$. On the other hand the monotonicity properties in Lemma 3.5, imply for $H \subset L$ the inequalities $q(L, H) \leq q(L, L) \leq q(H, L)$ or, alternatively, $\underline{q}(H, L) \leq \underline{q}(L, L) \leq \underline{q}(L, H)$. On combining the first, resp. the second set of inequalities, the assertion follows both ways.

Now we turn to further relationships between various capacities and Chebyshev constants. The following lemma is standard, we only state it for completeness and because we could not find a standard reference containing exactly the form we need.

Lemma 3.8. The convex combinations of Dirac measures $\delta_{x}$ concentrated on points $x$ of a given compact set $K \subset X$ form a weak*-dense subset of $\mathfrak{M}_{1}(K)$.

Proof. Denote $B$ the unit ball in the Banach space $\mathfrak{M}^{ \pm}(K)$. Writing 1 for the constant one function on $K$, we have $\mathfrak{M}_{1}(K)=\left\{\mu: \mu \in B, \int_{K} 1 \mathrm{~d} \mu=1\right\}$, so $\mathfrak{M}_{1}(K)$ is a weak*closed subset of $B$. Since $B$ is weak*-compact in view of the Banach-Alaoglu Theorem, $\mathfrak{M}_{1}(K) \subset B$ is weak ${ }^{*}$-compact, too.

It is well-known that the set $E$ of extremal points of $\mathfrak{M}_{1}(K)$ consists of the Dirac measures $\delta_{x}, x \in K$ (see, e.g., [23, Proposition 2.1.2, page 52]). Thus by the KreŭnMilman theorem (see, e.g., [34, Sec. 10.4]) the set of convex combinations conv $E$ of Dirac measures is weak ${ }^{*}$-dense in $\mathfrak{M}_{1}(K)$.

By the above lemma, given any measure $\mu \in \mathfrak{M}_{1}(K)$ we can approximate it in the weak*-topology by a finitely supported measure. On the other hand, finitely supported measures can further be approximated even in a more strict sense.

Lemma 3.9. For any finitely supported probability measure $\nu$ and $\varepsilon>0$, there exists a probability measure of the form $\mu=\frac{1}{m} \sum_{i=1}^{m} \delta_{z_{i}}$ having the same support as $\nu$ and satisfying $(1-\varepsilon) \nu \leq \mu \leq(1+\varepsilon) \nu$.

Proof. Let $\nu=\frac{1}{n} \sum_{j=1}^{n} \alpha_{j} \delta_{w_{j}}$ with the coefficients $\alpha_{j}$ being strictly positive. Choose $\beta_{j} \in\left((1-\varepsilon) \alpha_{j},(1+\varepsilon) \alpha_{j}\right) \cap \mathbb{Q}_{+}$such that $\sum_{j=1}^{n} \beta_{j}=1$. Define $\mu=\sum_{j=1}^{n} \beta_{j} \delta_{w_{j}}$, so for some $m \in \mathbb{N}$ and with an appropriate set of points $z_{i}$ (i.e., with possible repetitions of the points $\left.w_{j}\right) \mu$ can be written as $\mu=\frac{1}{m} \sum_{i=1}^{m} \delta_{z_{i}}$.

The lemma below is a well-known version of the Stone-Weierstraß Theorem. Actually, without any assumption on the coefficients $\alpha_{i}$, it is the usual approximation theorem used frequently in the literature. The proof of this slightly "stronger" statement goes along the same lines as that of the original version, see for example Bourbaki [3, Lemma III.1.2 \& Exercise III.1.6].

Lemma 3.10. Let $f \in C_{c}(X \times X)$ and $\varepsilon>0$. Then there exists $g \in C_{c}(X) \otimes C_{c}(X)$ of the form

$$
g(x, y)=\sum_{i=1}^{n} \alpha_{i} \cdot g_{i}(x) g_{i}^{\prime}(y)
$$


with $g_{i}, g_{i}^{\prime} \in C_{c}(X), 0 \leq g_{i}, g_{i}^{\prime} \leq 1$ and $\left|\alpha_{i}\right| \leq\|f\|$ satisfying

$$
\|f-g\| \leq \varepsilon .
$$

One can always take also $\alpha_{i} \in \mathbb{Q}$. Furthermore, in case $f \geq 0$ the coefficients $\alpha_{i}$ can be chosen from $\mathbb{Q}_{+}$.

Lemma 3.11. Let $K \subset X$ be compact and $k$ be continuous on $K \times K$. Then the mapping

$$
\mathfrak{M}_{1}(K) \ni \mu \mapsto U^{\mu}\left(=\int_{X} k(\cdot, y) \mathrm{d} \mu(y)\right)
$$

is continuous from the weak $k^{*}$-topology to the sup-norm topology of $C(K)$.

Proof. Fix $\mu \in \mathfrak{M}_{1}(K)$ and take $\nu \in \mathfrak{M}_{1}(K)$. Further, for $\left.k\right|_{K \times K}$ take $g(x, y)=\sum_{i=1}^{n} \alpha_{i}$. $g_{i}(x) g_{i}^{\prime}(y)$ supplied by Lemma 3.10. Then we have

$$
\begin{aligned}
& \int_{X} k(x, y) \mathrm{d} \mu(y)-\int_{X} k(x, y) \mathrm{d} \nu(y)=\int_{X} k(x, y) \mathrm{d} \mu(y)-\int_{X} g(x, y) \mathrm{d} \mu(y)+ \\
& \quad+\int_{X} g(x, y) \mathrm{d} \mu(y)-\int_{X} g(x, y) \mathrm{d} \nu(y)+\int_{X} g(x, y) \mathrm{d} \nu(y)-\int_{X} k(x, y) \mathrm{d} \nu(y) .
\end{aligned}
$$

So we obtain

$$
\begin{aligned}
& \left|\int_{X} k(x, y) \mathrm{d} \mu(y)-\int_{X} k(x, y) \mathrm{d} \nu(y)\right| \leq \\
& \quad \leq \varepsilon\|\mu\|+\left|\int_{X} g(x, y) \mathrm{d} \mu(y)-\int_{X} g(x, y) \mathrm{d} \nu(y)\right|+\varepsilon\|\nu\| .
\end{aligned}
$$

Using the particular form of $g$ we can write

$$
\begin{aligned}
\left|\int_{X} g(x, y) \mathrm{d} \mu(y)-\int_{X} g(x, y) \mathrm{d} \nu(y)\right|= \\
=\left|\sum_{i=1}^{n} \alpha_{i} \cdot g_{i}(x)\left(\int_{X} g_{i}^{\prime}(y) \mathrm{d} \mu(y)-\int_{X} g_{i}^{\prime}(y) \mathrm{d} \nu(y)\right)\right| \leq \\
\leq n\|k\| \max _{i=1, \ldots, n}\left|\int_{X} g_{i}^{\prime}(y) \mathrm{d} \mu(y)-\int_{X} g_{i}^{\prime}(y) \mathrm{d} \nu(y)\right| \leq \varepsilon,
\end{aligned}
$$

if $\nu$ is in an appropriate weak ${ }^{*}$-neighborhood of $\mu$. Combining this with (3.2) finishes the proof.

Theorem 3.12. Let $k$ be a continuous kernel. Then $q(H, K)=q^{\#}(H, K)$ for all $H \subset X$ and compact set $K \subset X$. 
Proof. Let us first prove $q\left(K^{\prime}, K\right)=q^{\#}\left(K^{\prime}, K\right)$ whenever $K^{\prime} \Subset X$ is compact. From definition $q\left(K^{\prime}, K\right) \leq q^{\#}\left(K^{\prime}, K\right)$ is trivial. Now if $q\left(K^{\prime}, K\right)=+\infty$, there is nothing to prove, hence let us assume that $q\left(K^{\prime}, K\right)<+\infty$. For an arbitrarily fixed $\varepsilon>0$ take $\mu$ a probability measure supported in $K^{\prime}: \operatorname{supp} \mu \subset K^{\prime}$ such that (by (2.2))

$$
Q(\mu ; K) \leq q\left(K^{\prime}, K\right)+\varepsilon
$$

Using Lemma 3.8 and Lemma 3.9 we find points $x_{j} \in K^{\prime}(j=1, \ldots, n, n \in \mathbb{N})$ such that the measure $\nu=\frac{1}{n} \sum_{i=1}^{n} \delta_{x_{i}}$ is close to $\mu$ (lies in an arbitrarily prescribed weak*neighborhood of $\mu$ ), so by Lemma 3.11 the estimate $|Q(\mu ; K)-Q(\nu ; K)| \leq \varepsilon$ holds. So $q^{\#}\left(K^{\prime}, K\right) \leq Q(\nu ; K) \leq q\left(K^{\prime}, K\right)+2 \varepsilon$, and taking infimum in $\varepsilon$ yields the statement for compact sets $K^{\prime}$. We conclude the proof by referring to Lemma 2.4.

\section{Dual Chebyshev constants}

Analogously to the dual formulations $q$ and $\underline{q}$, we define the dual Chebyshev constants. This will enable us to identify the rendezvous intervals.

Definition 4.1. For arbitrary $H, L \subset X$ the $n^{\text {th }}$ (general) dual Chebyshev constant of $L$ relative to $H$ is defined as

$$
\bar{M}_{n}(H, L):=\inf _{w_{1}, \ldots, w_{n} \in H} \sup _{x \in L} \frac{1}{n}\left(\sum_{j=1}^{n} k\left(x, w_{j}\right)\right) .
$$

Showing the quasi-monotonicity of $\bar{M}_{n}(H, L)$ and using Lemma 3.1 give immediately the following.

Proposition 4.2. The sequence $\bar{M}_{n}(H, L)$ of dual Chebyshev constants has limit.

Proof. Suppose that $\bar{M}_{n}(H, L)<+\infty$ for some $n \in \mathbb{N}$. This means that there are $x_{1}, x_{2}, \ldots, x_{n} \in H$ with

$$
\sup _{y \in L} \frac{1}{n} \sum_{i=1}^{n} k\left(x_{i}, y\right)<+\infty
$$

But then taking $x_{n+1}:=x_{n}$, we see that $\bar{M}_{n+1}(H, L)$ is also finite. Summing up, we see that the sequence $\bar{M}_{n}(H, L)$ is either constant $+\infty$ or eventually finite in which case Fekete's Lemma 3.1 is applicable, because $\bar{M}_{n}(H, L)$ is quasi-monotone decreasing. In both cases the limit exists.

Note that by quasi-monotonicity we have $\bar{M}_{n}(H, L) \geq \bar{M}(H, L)$ for $n \in \mathbb{N}$. The limit is denoted by $\bar{M}(H, L)$, and in particular by $\bar{M}(H)$.

It is easy to see that for arbitrary sets $H, L \subset X$ the $n^{\text {th }}$ Chebyshev constants and dual Chebyshev constants are just the lower, resp. upper endpoints of the intervals of the $n^{\text {th }}$ rendezvous sets (2.19) and rendezvous sets (2.20); and similarly with the energies $q(H, L)$ and dual energies $\underline{q}(H, L)$ regarding $(2.21)$. 
Proposition 4.3. For arbitrary subsets $H, L \subset X$ we have

$$
\begin{array}{ll}
\inf R_{n}(H, L)=M_{n}(H, L), & \sup R_{n}(H, L)=\bar{M}_{n}(H, L), \\
\inf R(H, L)=M(H, L), & \sup R(H, L)=\bar{M}(H, L), \\
\inf A(H, L)=\underline{q}(H, L), & \sup A(H, L)=q(H, L) .
\end{array}
$$

That is, we have

$$
\begin{aligned}
& R_{n}(H, L)=\left[M_{n}(H, L), \bar{M}_{n}(H, L)\right], \\
& R(H, L)=[M(H, L), \bar{M}(H, L)], \\
& A(H, L)=[\underline{q}(H, L), q(H, L)] .
\end{aligned}
$$

Proof. By Remark 2.12 all the intervals are intersections of certain families $\mathcal{F}$ of closed intervals. Hence the supremum (resp. infimum) of the lower (resp. upper) endpoints of the elements of $\mathcal{F}$ is the lower (resp. upper) endpoint of $\bigcap \mathcal{F}$.

Remark 4.4. It is important to note that intervals appearing in the proposition above may be empty. This occurs, for example for $R(H, L)$, if $\bar{M}(H, L)<M(H, L)^{2}$. Thus, for example, proving that the rendezvous interval is non-empty is the same as showing $M(H, L) \leq \bar{M}(H, L)$.

Proposition 4.5. For any $H, L \subset X$ the dual Chebyshev constant $\bar{M}(H, L)$ coincides with $q^{\#}(H, L)$. Also, the dual statement $M(H, L)=\underline{q}^{\#}(H, L)$ holds true.

Proof. The inequality $q^{\#}(H, L) \leq \bar{M}_{n}(H, L)$ is trivial since in the definition of $\bar{M}_{n}(H, L)$ we allow only a subclass of finitely supported probability measures. Hence $q^{\#}(H, L) \leq$ $\bar{M}(H, L)$. If $q^{\#}=+\infty$, we are ready. Otherwise fix $\eta>0$ arbitrarily and take $\nu=$ $\sum_{j=1}^{k} \alpha_{j} \delta_{x_{j}}, x_{j} \in H$ such that $Q(\nu ; L)<q^{\#}(H, L)+\eta$. For $\varepsilon>0$ Lemma 3.9 supplies a measure $\mu=\frac{1}{m} \sum_{i=1}^{m} \delta_{z_{i}} \in \mathfrak{M}_{1}(H)$ with

$$
Q(\mu ; L)=\sup _{y \in L} \frac{1}{m} \sum_{i=1}^{m} k\left(z_{i}, y\right) \leq \sup _{y \in L} \sum_{j=1}^{n}(1+\varepsilon) \alpha_{j} k\left(x_{j}, y\right) \leq(1+\varepsilon)\left(q^{\#}(H, L)+\eta\right) .
$$

Since this holds for all $\varepsilon, \eta>0$, the proof concludes. The case of $\underline{q}^{\#}$ is similar.

Corollary 4.6. For any $H \subset X$ one has $\underline{q}^{\#}(H)=M(H)$ and $q^{\#}(H)=\bar{M}(H)$.

\section{Chebyshev constant and energy}

The results presented in this section are complementary to those in Lemma 3.6. Indeed, the mentioned lemma gives $\underline{q}(H, L) \leq q(L, H)$, but we prove here equality under certain assumptions. The relation to $M(H, L)$ is also observed.

Already Fuglede [15] proves (even under more general assumptions) the relationship between the dual notions of energies.

\footnotetext{
${ }^{2}$ Here and also in the sequel, we use the convention $[a, b]=\emptyset$, whenever $b<a$.
} 
Remark 5.1. To prove $q(H, L)=q(L, H)$ obviously requires some minimax results. If our kernel was finite-valued, then a perfect tool would be Kneser's or Kassay and Kolumbán's minimax theorem. In the setting of rendezvous numbers, Thomassen applies the Neumann minimax theorem, Stadje refers to a more game-theoretically formulated minimax theorem in Ferguson [11]. Why is this confusing abundance of occurrences of various minimax results? Probably, the reason is best explained by Frenk, Kassay and Kolumbán in [13], who in fact show that all these and many other well-known minimax results (e.g., Ky-Fan, Kakutani) are (more or less elementarily) equivalent to each other. However, our kernel may take infinite values, thus we need a respective minimax theorem. A possible choice would be to refer to Glicksberg's apparently unfindable work [18], which is mentioned by Fuglede [15]. Actually, Fuglede tackles the problem by an approximation argument. Instead we choose to present our version with a full proof, which is based on the lecture notes of Pollard [29].

Let $C$ be a convex set in a linear space. A function $f: C \rightarrow(-\infty,+\infty]$ said to be convex, if

$$
f(\alpha x+(1-\alpha) y) \leq \alpha f(x)+(1-\alpha) f(y)
$$

the multiplication $0 \cdot f(x)$ is interpreted as 0 and $\alpha \cdot+\infty=+\infty, \alpha \neq 0$. Analogously one defines concavity.

Theorem 5.2 (Minimax Theorem). Let $A$ be a compact, convex subset of a Hausdorff topological vector space $U$ and $B$ be a convex subset of the linear space $V$. Let $k: A \times B \rightarrow$ $(-\infty,+\infty]$ be l.s.c. on $A$ for fixed $y \in B$, and assume that $k$ is convex in the first and concave in the second variable. Then

$$
\sup _{y \in B} \inf _{x \in A} k(x, y)=\inf _{x \in A} \sup _{y \in B} k(x, y) .
$$

Proof. The inequality " $\leq$ " is trivial. For the converse we may assume that the left hand side is finite, otherwise we are done. Let both $R>S$ be strictly greater than the left hand side. We have to show that the right hand side does not exceed $R$. For this purpose define $A_{y, r}:=\{x \in A: k(x, y) \leq r\}$ for $y \in B$ and $r \in \mathbb{R}$. By assumption on $R$ we have $A_{y, R} \neq \emptyset$ for all $y \in B$. Now we have to prove that also $\bigcap_{y \in B} A_{y, R} \neq \emptyset$. Indeed, if $x_{0}$ belongs to this intersection, then $\sup _{y \in B} k\left(x_{0}, y\right) \leq R$ and the claim follows.

So let us turn to proving the non-emptyness of the intersection. Since the sets $A_{y, R}$ are all compact (and non-empty), it suffices to prove that every finite family $A_{y_{i}, R}, i=1, \ldots, n$ $(n \in \mathbb{N})$ has non-empty intersection.

Let first $n=2$. We argue by contradiction, i.e., suppose that $A_{y_{1}, R} \cap A_{y_{2}, R}=\emptyset$. Introduce the notations $A^{\prime}:=\left\{x: x \in A, k\left(x, y_{1}\right)<+\infty, k\left(x, y_{2}\right)<+\infty\right\}$ and $A_{j}:=$ $A_{y_{j}, R} \cap A^{\prime}, j=1,2$. By assumption, all these sets are convex, too. We will first find an $\alpha \in[0,1]$ such that

$$
\alpha k\left(x, y_{1}\right)+(1-\alpha) k\left(x, y_{2}\right) \geq R \quad\left(\forall x \in A^{\prime}\right)
$$

If $x \notin A_{y_{1}, R} \cup A_{y_{2}, R}$, then the above inequality holds for all $\alpha \in[0,1]$. In case $x \in A_{1}$, we must choose $\alpha$ so that

$$
k\left(x, y_{2}\right)-R \geq \alpha\left(k\left(x, y_{2}\right)-k\left(x, y_{1}\right)\right) \quad\left(\forall x \in A_{1}\right)
$$


In view of the disjointness assumption $A_{y_{1}, R} \cap A_{y_{2}, R}=\emptyset$ we have for any $x \in A_{y_{1}, R}$ $k\left(x, y_{2}\right)>R \geq k\left(x, y_{1}\right)$, hence we get the equivalent form

$$
(1 \geq) \inf _{x \in A_{1}} \frac{k\left(x, y_{2}\right)-R}{k\left(x, y_{2}\right)-k\left(x, y_{1}\right)} \geq \alpha
$$

Similarly, for the case $x \in A_{2}$ the constraint on $\alpha$ will be $\alpha\left(k\left(x, y_{1}\right)-k\left(x, y_{2}\right)\right) \geq R-$ $k\left(x, y_{2}\right)\left(\forall x \in A_{2}\right)$, that is

$$
\alpha \geq \sup _{x \in A_{2}} \frac{R-k\left(x, y_{2}\right)}{k\left(x, y_{1}\right)-k\left(x, y_{2}\right)}(\geq 0)
$$

Note that by the assumption of disjointness, the two constraints are independent, thus $\alpha$ can be chosen satisfying both requirements if and only if

$$
\frac{R-k\left(x_{2}, y_{2}\right)}{k\left(x_{2}, y_{1}\right)-k\left(x_{2}, y_{2}\right)} \leq \frac{k\left(x_{1}, y_{2}\right)-R}{k\left(x_{1}, y_{2}\right)-k\left(x_{1}, y_{1}\right)} \quad\left(\forall x_{1} \in A_{1} \quad \text { and } \quad \forall x_{2} \in A_{2}\right)
$$

As the denominators are positive, some computation leads to the equivalent formulation

$$
\left(R-k\left(x_{2}, y_{2}\right)\right)\left(R-k\left(x_{1}, y_{1}\right)\right) \leq\left(k\left(x_{1}, y_{2}\right)-R\right)\left(k\left(x_{2}, y_{1}\right)-R\right) \quad\left(x_{j} \in A_{j}, j=1,2\right) .
$$

So let $x_{1} \in A_{1}$ and $x_{2} \in A_{2}$ be arbitrary. Note that the terms on the right hand side are necessarily positive in view of the choice of $x_{1}, x_{2}$ and the assumption of disjointness. So we may assume that also on the left hand side of (5.2) both terms are strictly positive. Then there exists $\theta \in(0,1)$ with

$$
\theta k\left(x_{1}, y_{1}\right)+(1-\theta) k\left(x_{2}, y_{1}\right)=R .
$$

Define $x_{\theta}=\theta x_{1}+(1-\theta) x_{2}$ for this particular $\theta$. By convexity $k\left(x_{\theta}, y_{1}\right) \leq R$, so $x_{\theta} \in A_{y_{1}, R}$ and therefore $x_{\theta} \notin A_{y_{2}, R}$. On the other hand

$$
+\infty>\theta k\left(x_{1}, y_{2}\right)+(1-\theta) k\left(x_{2}, y_{2}\right) \geq k\left(x_{\theta}, y_{2}\right)>R
$$

Combining (5.3) and (5.4) we arrive at

$$
\frac{R-k\left(x_{1}, y_{1}\right)}{k\left(x_{2}, y_{1}\right)-R}=\frac{1-\theta}{\theta}<\frac{k\left(x_{1}, y_{2}\right)-R}{R-k\left(x_{2}, y_{2}\right)}
$$

which gives (5.2).

Suppose that incidentally $\alpha=1$. Then

$$
k\left(x, y_{1}\right) \geq R
$$

holds for all $x \in A^{\prime}$. Note that $M:=\inf _{x \in A} k\left(x, y_{2}\right)$ is a finite minimum value because of the lower semicontinuity of $k$ and the compactness of $A$. Now let $\beta:=(S-M) /(R-M)$ and $y_{0}:=\beta y_{1}+(1-\beta) y_{2}$. By concavity of $k$ in the second variable, we find

$$
k\left(x, y_{0}\right) \geq \beta k\left(x, y_{1}\right)+(1-\beta) k\left(x, y_{2}\right) \geq \begin{cases}+\infty, & \text { if } x \notin A^{\prime} \\ \beta R+(1-\beta) M=S, & \text { if } x \in A^{\prime}\end{cases}
$$


Hence in both cases $k\left(x, y_{0}\right) \geq S$. It follows that $\inf _{x \in A} k\left(x, y_{0}\right) \geq S$, a contradiction with the choice of $S$. Therefore, we conclude that $\alpha=1$ is not possible.

Similarly we find that $\alpha=0$ can not hold. So we obtain that there is $\alpha \in(0,1)$ satisfying (5.1). But then, actually, for all $x \in A$, since $\alpha \in(0,1)$. By the concavity of $k$ this implies

$$
\inf _{x \in A} k\left(x, \alpha y_{1}+(1-\alpha) y_{2}\right) \geq R,
$$

which, in view of $\alpha y_{1}+(1-\alpha) y_{2} \in B$ would be a contradiction to the choice of $R$.

For completing the proof, we apply induction. Let $n \geq 2$ and assume that the assertion has already been proved for $n$. Then replacing $A$ by the compact, convex set $A_{y_{n+1}, R}$ and considering the sets $A_{y_{m}, R} \cap A_{y_{n+1}, R}, m=1, \ldots, n$, we can deduce $\bigcap_{m=1}^{n+1} A_{y_{m}, R} \neq \emptyset$, too. This concludes the proof.

Now we are in the position to investigate the relationship between $q$ and $q$.

Theorem 5.3 (Fuglede). Let $k$ be any symmetric, non-negative kernel, $K \Subset X$ compact and $L \subset X$ be any subset. Then the equality

$$
\begin{aligned}
q(K, L) & =\inf _{\mu \in \mathfrak{M}_{1}(K)} \sup _{\nu \in \mathfrak{M}_{1}(L)} \int_{L} \int_{K} k(x, y) \mathrm{d} \mu(y) \mathrm{d} \nu(x)= \\
& =\sup _{\nu \in \mathfrak{M}_{1}(L)} \inf _{\mu \in \mathfrak{M}_{1}(K)} \int_{L} \int_{K} k(x, y) \mathrm{d} \mu(y) \mathrm{d} \nu(x)
\end{aligned}
$$

holds true. Furthermore, one has $q(K, L)=\underline{q}(L, K)$.

Proof. Obviously

$$
\begin{aligned}
q(K, L) & =\inf _{\mu \in \mathfrak{M}_{1}(K)} \sup _{\substack{\nu=\delta_{z} \\
z \in L}} \int_{L} \int_{K} k(x, y) \mathrm{d} \mu(y) \mathrm{d} \nu(x) \leq \\
& \leq \inf _{\mu \in \mathfrak{M}_{1}(K)} \sup _{\nu \in \mathfrak{M}_{1}(L)} \int_{L} \int_{K} k(x, y) \mathrm{d} \mu(y) \mathrm{d} \nu(x)
\end{aligned}
$$

holds. Observe that $A:=\mathfrak{M}_{1}(K)$ is a convex, nonempty, and weak*-compact subset of $U:=\mathfrak{M}^{ \pm}(K)$, while $B:=\mathfrak{M}_{1}(L)$ is a convex, nonempty subset of the vector space $V:=\mathfrak{M}^{ \pm}(X)$; moreover, the mapping

$$
f: A \times B \rightarrow \mathbb{R} \quad f(\mu, \nu):=\int_{L} \int_{K} k(x, y) \mathrm{d} \mu(y) \mathrm{d} \nu(x)
$$

is affine on $A \times B$ (in fact linear on $U \times V$ ), while for any fixed measure $\nu \in B:=\mathfrak{M}_{1}(L)$ it is lower semicontinuous in $\mu \in A:=\mathfrak{M}_{1}(K)$ by Lemma $\left.2.3 \mathrm{~b}\right)$. Hence we can continue 
(5.5) by an application of the Minimax Theorem 5.2 to obtain

$$
\begin{aligned}
q(K, L) & \leq \inf _{\mu \in \mathfrak{M}_{1}(K)} \sup _{\nu \in \mathfrak{M}_{1}(L)} \int_{L} \int_{K} k(x, y) \mathrm{d} \mu(y) \mathrm{d} \nu(x)= \\
& =\sup _{\nu \in \mathfrak{M}_{1}(L)} \inf _{\mu \in \mathfrak{M}_{1}(K)} \int_{L} \int_{K} k(x, y) \mathrm{d} \mu(y) \mathrm{d} \nu(x)= \\
& =\sup _{\nu \in \mathfrak{M}_{1}(L)} \inf _{\mu \in \mathfrak{M}_{1}(K)} \int_{L} U^{\mu}(x) \mathrm{d} \nu(x) \leq \sup _{\nu \in \mathfrak{M}_{1}(L)} \inf _{\mu \in \mathfrak{M}_{1}(K)} \sup _{x \in L} U^{\mu}(x)= \\
& =\inf _{\mu \in \mathfrak{M}_{1}(K)} Q(\mu ; L)=q(K, L) .
\end{aligned}
$$

Thus all expressions in (5.6) must be equal.

By Lemma 3.6 and by what we proved in (5.6)

$$
\begin{aligned}
\underline{q}(L, K) & \leq q(K, L)=\sup _{\nu \in \mathfrak{M}_{1}(L)} \inf _{\mu \in \mathfrak{M}_{1}(K)} \int_{L} \int_{K} k(x, y) \mathrm{d} \mu(y) \mathrm{d} \nu(x)= \\
& =\sup _{\nu \in \mathfrak{M}_{1}(L)} \inf _{\mu \in \mathfrak{M}_{1}(K)} \int_{K} U^{\nu}(y) \mathrm{d} \mu(y) \leq \sup _{\nu \in \mathfrak{M}_{1}(L)} \inf _{y \in K} U^{\nu}(y)= \\
& =\sup _{\nu \in \mathfrak{M}_{1}(L)} \underline{Q}(\nu ; K)=\underline{q}(L, K),
\end{aligned}
$$

where the last inequality follows because $\delta_{y} \in \mathfrak{M}_{1}(K)$ whenever $y \in K$. This concludes the proof.

Among other related things, and even under more general assumptions, Ohtsuka shows also the following results [26]. Notice that again the symmetry of the kernel is actually not important.

Theorem 5.4 (Ohtsuka). Let $k$ be any symmetric l.s.c. kernel, $L$ be an arbitrary and $K$ be a compact subset of $X$. Then the equality $M(L, K)=\underline{q}(L, K)$ holds.

Proof. Obviously $M(L, K) \leq \underline{q}(L, K)$ holds. First let us prove the converse inequality in case $L$ is compact, too. Take $\bar{\nu}_{0} \in \mathfrak{M}_{1}(L)$ arbitrarily. Using Lemma 3.8 and Lemma 3.9, we can select a net of measures $\nu_{\alpha}:=\frac{1}{n_{\alpha}} \sum_{i=1}^{n_{\alpha}} \delta_{w_{i}}^{\alpha}, w_{i} \in L$ weak ${ }^{*}$-converging to $\nu_{0}$.

Then by Lemma $2.3 \mathrm{~b}$ ), we have for each $\alpha$ a measure $\mu_{\alpha} \in \mathfrak{M}_{1}(K)$ such that

$$
\int_{L} \int_{K} k(x, y) \mathrm{d} \mu_{\alpha}(x) \mathrm{d} \nu_{\alpha}(y)=\inf _{\mu \in \mathfrak{M}_{1}(K)} \int_{L} \int_{K} k(x, y) \mathrm{d} \mu(x) \mathrm{d} \nu_{\alpha}(y) .
$$

Again by weak*-compactness we may assume that $\mu_{\alpha}$ converges to some $\mu_{0} \in \mathfrak{M}_{1}(K)$, and again by lower semicontinuity we find that

$$
\int_{L} \int_{K} k(x, y) \mathrm{d} \mu_{0}(x) \mathrm{d} \nu_{0}(y) \leq \liminf \int_{\alpha} \int_{K} k(x, y) \mathrm{d} \mu_{\alpha}(x) \mathrm{d} \nu_{\alpha}(y) .
$$


These last two relations imply

$$
\begin{aligned}
& \inf _{\mu \in \mathfrak{M}_{1}(K)} \int_{L} \int_{K} k(x, y) \mathrm{d} \mu(x) \mathrm{d} \nu_{0}(y) \leq \int_{L} \int_{K} k(x, y) \mathrm{d} \mu_{0}(x) \mathrm{d} \nu_{0}(y) \leq \\
& \leq \liminf _{\alpha} \inf _{\mu \in \mathfrak{M}_{1}(K)} \int_{L} \int_{K} k(x, y) \mathrm{d} \mu(x) \mathrm{d} \nu_{\alpha}(y)= \\
& =\liminf _{\alpha} \inf _{\mu \in \mathfrak{M}_{1}(K)} \int_{K} \frac{1}{n_{\alpha}} \sum_{i=1}^{n_{\alpha}} k\left(x, w_{i}^{\alpha}\right) \mathrm{d} \mu(x) \leq \\
& \leq \liminf _{\alpha} \inf _{\mu=\delta_{z}} \int_{z \in K} \frac{1}{n_{\alpha}} \sum_{i=1}^{n_{\alpha}} k\left(x, w_{i}^{\alpha}\right) \mathrm{d} \mu(x)= \\
& =\liminf _{\alpha} \inf _{z \in K} \frac{1}{n_{\alpha}} \sum_{i=1}^{n_{\alpha}} k\left(z, w_{i}^{\alpha}\right) \leq \liminf _{\alpha} M_{n_{\alpha}}(L, K)=M(L, K) .
\end{aligned}
$$

Taking supremum in $\nu_{0} \in \mathfrak{M}_{1}(L)$ we obtain

$$
\sup _{\nu \in \mathfrak{M}_{1}(L)} \inf _{\mu \in \mathfrak{M}_{1}(K)} \int_{L} \int_{K} k(x, y) \mathrm{d} \nu(y) \mathrm{d} \mu(x) \leq M(L, K) .
$$

The left-hand side of $(5.7)$ equals $q(K, L)=\underline{q}(L, K)$ by Theorem 5.3 , hence the assertion follows in case $L$ is compact.

Now let $\emptyset \neq L \subset X$ be arbitrary. Let $\nu_{0} \in \mathfrak{M}_{1}(L)$ be with $\operatorname{supp} \nu_{0} \Subset L$. Then we have

$$
\underline{Q}\left(\nu_{0} ; K\right) \leq \underline{q}\left(\operatorname{supp} \nu_{0}, K\right)=M\left(\operatorname{supp} \nu_{0}, K\right) \leq M(L, K),
$$

where we used what we had proved above for the compact case together with Lemma 3.5. Now applying (2.15) from Lemma 2.7 concludes the proof.

\section{Rendezvous numbers}

Before drawing some consequences of the above results, let us summarize them as follows.

Corollary 6.1. Let $H, L \subset X$, then

$$
M(H, L)=\underline{q}^{\#}(H, L) \leq \underline{q}(H, L) \leq q(L, H) \leq q^{\#}(L, H)=\bar{M}(L, H) .
$$

If $L \subset X$ is compact, then

$$
M(H, L)=\underline{q}^{\#}(H, L)=\underline{q}(H, L)=q(L, H) .
$$

If $K \subset X$ is compact and $k$ is continuous, then

$$
q(L, K)=q^{\#}(L, K)=\bar{M}(L, K) .
$$


Let us shortly comment on the above proved (in)equalities. This is not closely related to rendezvous numbers, but complements the abstract potential theoretic point of view.

Remark 6.2. We say that the kernel $k$ satisfies the maximum principle if for every measure $\mu \in \mathfrak{M}_{1}$

$$
U^{\mu}(x) \leq \sup _{y \in \operatorname{supp} \mu} U^{\mu}(y) \quad \text { for all } x \in X
$$

Note that if the kernel $k$ has the maximum principle then $w(H)=v(H)=u(H)$ as discussed in [14], but since $w(H) \leq q(H) \leq u(H)$, the energy $q(H)$ equals the other ones, too.

In [8] it is shown that assuming the maximum principle for the kernel implies $D(K)=$ $w(K)=u(K)=M(K)$ for all $K \subset X$ compact set. In this case we also have $w(K)=$ $q(K)=u(K)=M(K)$. Without assuming the maximum principle, in general we only know $M(K)=q(K)$ by Equality (6.2) in the above corollary. Keeping in mind Theorem 3.4, we see that indeed the maximum principle implies the equality $D(K)=M(K)$. Recall that, in the classical case of the logarithmic kernel $k(x, y)=-\log |x-y|$ on $\mathbb{C}$, the equality of the two Chebyshev constants $C(K)=M(K)$ holds. Indeed, as proved above $M(K)=M(K, K)=q(K)$ and $C(K)=M(\mathbb{C}, K)=q(K, \mathbb{C})=u(K)$. So again the maximum principle is the reason for $M(K)=C(K)$.

Let us start with a result showing that our definitions for rendezvous intervals are non-trivial.

Theorem 6.3. Let $X$ be a locally compact Hausdorff space, $\emptyset \neq H \subset L \subset X$ be arbitrary, and let $k$ be any nonnegative, symmetric kernel on $X$. Then the intervals $R_{n}(H, L)$, $R(H, L)$ and $A(H, L)$ are nonempty.

Proof. It suffices to prove the assertion concerning $A(H, L)$ since it is contained in the other two sets (cf. Remark 2.12). By Proposition 4.3, $A(H, L)=[q(H, L), q(H, L)]$ holds, and the assertion reduces to stating $q(H, L) \leq q(H, L)$, which is furnished by Corollary 3.7 or (6.1) (cf. also Remark 4.4).

Remark 6.4. It is easy to see that in case $k: \Delta \rightarrow\{+\infty\}$ (where $\Delta$ is the diagonal $\{(x, x): x \in X\}$ ) we have $q^{\#}(H)=+\infty$, hence the rendezvous intervals $R(H)$ always extend to $+\infty$. Note also that in general $q(H) \neq+\infty$, providing examples where $A(H) \varsubsetneqq$ $R(H)$, and in particular, that $R(H)$ is not unique.

Example 6.5. Take, e.g., the case of $X:=H:=[0,1]$ and $k(x, y):=-\log |x-y|$, where obviously $\bar{M}(H)=q^{\#}(H)=+\infty$, while $M(H)=\log 4$, since we know that classical capacity of $[0,1]$ is $1 / 4$ (see, e.g., [32, Cor. 5.2.4]). Further $q(H)=\log 4$ holds also, because $w([0,1]) \leq M([0,1]) \leq q([0,1]) \leq u([0,1])$, but, as well-known, $k$ has maximum principle, so $w=q=u$.

Remark 6.6. It is easy to construct examples, when the rendezvous intervals are "almost empty": consider, e.g., $R_{n}(\mathbb{R}, \mathbb{R})=\{+\infty\}$. This and Remarks 2.13 and 2.14 explain the slightly disturbing situation that some papers state that "there is no rendezvous number" for cases where we find one. However, not only $+\infty$ can show up in the closure of intervals for the definition of rendezvous numbers, hence not only $+\infty$ can be a rendezvous number for us while does not exist for other authors. For the case of the $\ell_{p}$ spaces see [10]. 
Theorem 6.7. Let $X$ be any locally compact Hausdorff topological space, $k$ be any l.s.c., nonnegative, symmetric kernel function, and $\emptyset \neq K \Subset X$ compact. Then $A(K)$ consists of one single point. Furthermore, if $k$ is continuous, then even $R(K)$ consists of only one point.

Proof. The first part follows directly from Proposition 4.3, (4.1) and taking $L=K$ in the second part of Theorem 5.3 or in (6.2) in Corollary 6.1. For the second assertion, after taking $L=K$ both (6.2) and (6.3) in Corollary 6.1 can be applied, hence the assertion follows.

Example 6.8. Uniqueness for $R(K, L)$ (or $A(K, L)$ ) can not be obtained in general, even if $K \subset L$ and both are compact. Take, for example, $X:=\{a, b\}$ a discrete topological space with any kernel $k$ and $K:=\{a\}$. Then

$$
R(K, X)=[\min \{k(a, a), k(a, b)\}, \max \{k(a, a), k(a, b)\}],
$$

which reduces to one point only if $k(a, \cdot)$ is constant.

Theorem 6.9. If the kernel $k$ is continuous and $L$ is compact we have the equality $R(H, L)=A(H, L)$ for all $H \subset X$.

Proof. Proposition 4.3 identifies the rendezvous intervals and the results summarized in Corollary 6.1 conclude the proof.

Remark 6.10. The rendezvous set $R(H, L)$ is independent of any topology, while the notion of $A(H, L)$ essentially refers to regular Borel measures. Thus in some cases equality of them is useful.

\section{Concluding remarks and hints of further work}

The modified Definitions 2.20 and 2.21 of rendezvous numbers and average numbers lead to quite general existence results, well over the restrictions usual in the theory. The reason is the use of closure: in many cases, e.g., in cases when the kernel is not continuous, but only l.s.c., considering closure saves the day. Basic results of the theory of rendezvous numbers extend quite well in the new setting. For concrete results and also as regards heuristic ideas and general perception of the topic, general potential theory with a l.s.c. kernel on a locally compact, Hausdorff space turns out to be the relevant setting.

Given the above state of the matter, we aimed at understanding the working force and the general principles behind further, particular results. In metric spaces, there is a theory of rendezvous numbers related to invariant measures [22] and in relation to maximal energy $[4,39]$. These results - even if not the available proofs! - can all be conveniently described by potential theory, hence it is natural to expect general versions of the results known so far. For these see [9].

As for Banach spaces, extension of the existence and uniqueness results from locally compact spaces even to infinite dimensional normed spaces, deserves attention. We can accomplish this, showing that the new definition works definitely better than the old one 
of, say, strong rendezvous numbers without use of closure. One can describe a few further, fairly general results on rendezvous numbers of normed spaces, and therefore computations of rendezvous sets and numbers of concrete normed spaces become accessible. All this is most interesting in cases where up to now general understanding stopped at the fact that (strong) rendezvous numbers do not exist. Our results regarding the above questions will be presented in the forthcoming work [10].

\section{Acknowledgements}

For fruitful discussions, comments and references at the outset of this work, we are indebted to Norm Levenberg. We owe particular gratitude to Natalia Zorii for calling our attention to [26], which finally led us to realize that much of what we constructed on our way to describe the rendezvous numbers, had already been made ready in general linear potential theory by Fuglede and Ohtsuka. We also thank the anonymous referee for a careful reading and several valuable suggestions. We strongly hope that the thorough revision due to these references and suggestions improved the presentation of the material.

\section{References}

[1] V. Anagnostopoulos, Sz. Gy. Révész, Polarization constants for products of linear functionals over $\mathbb{R}^{2}$ and $\mathbb{C}^{2}$ and Chebyshev constants of the unit sphere, Publ. Math. Debrecen, 68 (1-2) (2006), 75-83, to appear.

[2] M. Baronti, E. Casini, P. L. PApini, On average distances and the geometry of Banach spaces, Nonlinear Anal., Theory Methods Appl. 42A (2000), no. 3, 533-541.

[3] N. Bourbaki, Éléments de Mathématique, XIII. Integration Ch.1,2,3 et 4., Hermann, Paris, 1965.

[4] G. BJÖRCK, Distributions of positive mass, which maximize a certain generalized energy integral, Ark. Mat. 3 (1958), 255-269.

[5] L. Carleson, Selected Problems on Exceptional Sets, Van Nostrand Mathematical Studies, No. 13 D. Van Nostrand Co., Inc., Princeton, N.J.-Toronto, Ont.-London 1967.

[6] G. Choquet, Diamètre transfini et comparaison de diverses capacités, Séminaire de Théorie du Potentiel, Faculté des Sciences de Paris, 1958/59, 7 pages.

[7] J. M. Cleary, S. A. Morris, D. Yost, Numerical geometry - numbers for shapes, Amer. Math. Monthly 93 (1986), 260-275.

[8] B. Farkas, B. Nagy, Transfinite diameter, Chebyshev constant, and capacity on locally compact spaces, Alfréd Rényi Institute preprint series, Hung. Acad. Sci., 7/2004, 10 pages. 
[9] B. FArkas, Sz. Gy. RÉvÉsz, Rendezvous numbers of metric spaces - a potential theoretic approach, Arch. Math., to appear.

[10] B. Farkas, Sz. Gy. RÉvész, Rendezvous numbers in normed spaces, Bull. Austr. Math. Soc. 72 (2005), 423-440, to appear.

[11] T.S. Ferguson, Mathematical Statistics. A Decision Theoretic Approach, Probability and Mathematical Statistics, Vol. 1, Academic Press, New York, London, 1967.

[12] M. Fekete, Über die Verteilung der Wurzeln bei gewissen algebraischen Gleichungen mit ganzahligen Koeffizienten, Math. Z. 17 (1923), 228-249.

[13] J. B. G. Frenk, G. Kassay, J. Kolumbán, On equivalent results in minimax theory, European J. Oper. Res., 157, (2004), no. 1, 46-58.

[14] B. Fuglede, On the theory of potentials in locally compact spaces, Acta Math. 103 (1960), 139-215.

[15] B. Fuglede, Le théorème du minimax et la théorie fine du potentiel, Ann Inst. Fourier 15 (1965), 65-87.

[16] J. C. García-Vázquez, R. Villa, The average distance property of the spaces $\ell_{\infty}^{n}(\mathbb{C})$ and $\ell_{1}^{n}(\mathbb{C})$, Arch. Math. 76 (2001), 222-230.

[17] O. Gross, The rendezvous value of a metric space, in: Advances in Game Theory, Ann. of Math. Studies, 52, Princeton, 1964, 49-53.

[18] I. L. Glicksberg, Minimax theorem with upper and lower semi-continuous payoffs, Rand. Corp. Res. Memorandum RM 478 (1950).

[19] H. Kneser, Sur un théoréme fondamental de la théorie des jeux, Comptes Rendus Acad. Sci. Paris 234 (1952), 2418-2420.

[20] N. S. LANDKOF, Foundations of modern potential theory, Die Grundlehren der mathematischen Wissenschaften, Band 180. Springer-Verlag, New York-Heidelberg, 1972.

[21] P. K. Lin, The average distance property of Banach spaces, Arch. Math. 68 (1997), 496-502.

[22] S. A. Morris, P. Nickolas, On the average distance property of compact connected metric spaces, Arch. Math. 40(1983), 459-463.

[23] P. Meyer-Nieberg, Banach lattices, Springer-Verlag, 1991.

[24] P. Nickolas, D. Yost, The average distance property for subsets of euclidean spaces, Arch. Math. 50 (1988), 380-384.

[25] M. Ohtsuka, On potentials in locally compact spaces, J. Sci. Hiroshima Univ. ser A 25, 135-352, 1961. 
[26] M. Ohtsuka, On various definitions of capacity and related notions, Nagoya Math. J. 30 (1967) 121-127.

[27] M. Ohtsuka, An application of the minimax theorem to the theory of capacity, $J$. Sci. Hiroshima Univ. ser A 29, 217-221, 1965.

[28] M. Ohtsuka, Generalized capacity and duality theorem in linear programming, $J$. Sci. Hiroshima Univ. ser A 30, 45-56, 1966.

[29] D. Pollard, The Minimax Theorem, Unpublished lecture notes, Paris, 2001. http://www.stat.yale.edu/ pollard/Paris2001/lectures.html

[30] Gy. Pólya, G. Szegő, Über den transfiniten Durchmesser (Kapazitätskonstante) von ebenen und räumlichen Punktmengen, J. Reine Angew. Math. 165 (1931), 4-49.

[31] Gy. Pólya, G. Szegő, Problems and Excercises in Analysis, vol. I, Die Grundlehren der matehmatischen Wissenschaften in Einzeldarstellungen, Bd. 193, Springer Verlag, 1972.

[32] T. Ransford, Potential Theory in the Complex Plane, London Mathematical Society Student Texts 28, Cambridge University Press, 1994.

[33] Sz. Gy. RÉvÉsz, Y. Sarantopoulos, Plank problems, polarization, and Chebyshev constants, J. Korean Math. Soc., 41 (2004) no. 1, 157-174.

[34] H. H. Schaefer, Topological Vector Spaces, Graduate Texts In Mathematics, vol. 3, Springer-Verlag, 1980.

[35] W. Stadje, A property of compact, connected spaces, Arch. Math. 36 (1981), $275-280$.

[36] C. Thomassen, The rendezvous number of a symmetric matrix and a compact connected metric space, Amer. Math. Monthly 107 (2000), no. 2, 163-166.

[37] R. Wolf, On the average distance property in finite dimensional real Banach spaces, Bull. Austral. Math. Soc. 51 (1994), 87-101.

[38] R. Wolf, On the average distance property of spheres in Banach spaces, Arch. Math. 62 (1994), 338-344.

[39] R. Wolf, On the average distance property and certain energy integrals, Ark. Mat. 35 (1997), 387-400.

[40] L. YAnG, J. Z. Zhang, Average distance constants of some compact convex spaces, L. China Univ. Sci. Tech. 17(1987), no. 1, 17-24.

[41] V. P. Zaharjuta, Transfinite diameter, Chebishev constants, and capacity for compacta in $\mathbb{C}^{n}$, Math. USSR Sbornik, vol. 25 (1975), no. 3, 350-364 (English translation). 